\title{
Diagnosis and Treatment of Postthrombotic Syndrome
}

\author{
Guido Bruning ${ }^{1}$ Jasmin Woitalla-Bruning ${ }^{1}$ Anne-Caroline Queisser ${ }^{1}$ Johanna Katharina Buhr ${ }^{1}$ \\ ${ }^{1}$ Department of Vein and Dermatosurgery, Tabea Hospital GmbH and \\ Co KG, Hamburg, Hamburg, Germany \\ Hämostaseologie 2020;40:214-220. \\ Address for correspondence Dr. med. Guido Bruning, Head of \\ Departement of Vein and Dermatosurgery, KrankenhausTabea $\mathrm{GmbH}$ \\ andCo KG, Kösterbergstraße 32, 22587 Hamburg, Germany \\ (e-mail: GBruning@tabea-krankenhaus.de).
}

\begin{abstract}
Keywords

- postthrombotic syndrome

- chronic venous insufficiency

- leg ulcer

- treatment

- diagnosis
\end{abstract}

After acute treatment of deep vein thrombosis, not only the risk but also associated side effects of postthrombotic syndrome (PTS) are often underestimated.

There are essentially two main types of PTS.

1. Obstructive type-no sufficient recanalization of the deep vein.

2. Refluctive type-sufficient recanalization of the deep vein, but insufficient venous valves in conjunction with venous reflux.

A statement regarding deep vein recanalization and venous valve function can be made at the earliest after 6 months.

PTS is often diagnosed without appropriate medical history. However, the assessment of the degree of recanalization and venous reflux is paramount to the medical prognosis. In our opinion, beside proximal thrombosis, sufficient recanalization combined with a strong venous reflux, especially in the popliteal vein, works as a powerful predictor for an unfavorable and fast progression of PTS and chronic venous insufficiency. Thus, the obstructive type is prognostically more favorable. For PTS in general, consistent compression therapy represents the first-line treatment option. With concomitant varicosis, one should assess whether the varicose veins represent primary varicosis with reflux or secondary varicosis without reflux. Especially in the presence of venous ulcers, the elimination of concomitant primary varicosis leads to an improved prognosis. Moist wound treatment is considered to be the standard treatment for all wounds undergoing secondary healing. A standardized set of topical therapeutic agents also facilitates the treatment. In individual cases "ulcershaving" and mesh graft transplantation proved to be successful.

\section{Introduction}

Postthrombotic syndrome (PTS) can follow acute deep vein thrombosis (DVT) despite an adequate first-line therapy in accordance with standard guidelines. ${ }^{1-3}$

The prevalence of PTS after a first time thrombosis is approximately $25 \%$ according to the data from the TULIPA registry. ${ }^{4}$ The severity of PTS can be rated using the Villalta score ( - Table 1 ), where PTS is conformed at a score of 5 points or above. ${ }^{5}$ Only in $1.5 \%$ of all cases a severe PTS (Villalta score $>15$ ) occurred after a first-time DVT. ${ }^{6}$ So far, however, a significant reduction of this number has not been achieved by an early recanalization procedure. ${ }^{7}$ By definition, PTS can only be diagnosed if signs of chronic venous insufficiency (CVI) are present after the occurrence of a DVT, including at least swelling and edema. Without both of those factors present, PTS cannot be diagnosed. Crucial for both the diagnosis of PTS and its prognosis are duplex sonographic results as well as existing risk factors for the development of severe PTS, such as proximal thrombosis, obesity, and CVI. ${ }^{8}$

PTS can result from the lack of recanalization of the affected veins or from the destruction of the valves in the affected venous sections. The development of PTS after DVT can be underestimated in its severity and prevalence by the physician received

October 17, 2019

accepted after revision

February 25, 2020 (c) 2020 Georg Thieme Verlag KG Stuttgart · New York
DOI https://doi.org/

10.1055/a-1145-0108. ISSN 0720-9355. 
Table 1 Villaltascore for postthromboticsyndrome

\begin{tabular}{|l|l|}
\hline Symptoms & Clinical signs \\
\hline Pain & Pretibial edema \\
\hline Cramps & Skin induration \\
\hline Heaviness & Hyperpigmentation \\
\hline Paresthesia & Redness \\
\hline Pruritus & Venous ectasia \\
\hline & Pain on calf compression \\
\hline
\end{tabular}

Note: 0 point $=$ absent, 1 point $=$ mild, 2 points $=$ moderate, 3 points $=$ severe, $5-9$ points $=$ mild, $10-14$ points $=$ moderate, $\geq 15$ points $=$ severe

treating the acute DVT, but also and mostly by the patient himself. The severity of CVI is rated using the CEAP (clinicaletiological-anatomical-pathophysiological) classification, ${ }^{9}$ which includes the cause of CVI (obstructive vs. reflux) as well as the affected venous segments ( - Table 2 ). Older classifications such as the Widmer classification are commonly in use but not as specific as the CEAP classification.

Hereinafter, an attempt is made to bring diagnostic tools and treatment options into an appropriate structure for patients and practitioners.

\section{Follow-up Periods}

The first follow-up examination after an acute DVT should only occur as soon as a therapy modification is in order, i.e., changing the dosage or drugs, or after 3 months with isolated lower leg thrombosis. We recommend this approach because in our professional opinion, despite the results of the duplex examination, no changes would be made to the current treatment plan either way. Usually a follow-up examination only makes sense after 6 months, as compression therapy is often only modified after this period of time. After the initial compression therapy, which should extend beyond the venous segment affected by DVT over a period of 6 months, further compression of the lower leg alone is usually sufficient. The intensity and duration of compression therapy should be recommended individually, since compression therapy should be adapted to the size of the leg, remaining destructions in the deep veins, and the wishes and compliance of the patient. Permanent compression therapy after DVT without subsequent PTS (i.e., after an isolated distal thrombosis or full reopening without valve insufficiency) is often not required. Within the SOX study, there were doubts whether permanent compression therapy could be useful for patients with PTS. Unfortunately, an exact conclusion cannot be made due to the lack of compliance of the collective wearing the compression stockings. In comparison, European studies could show a benefit for PTS patients who wore stockings. ${ }^{10-12}$

The degree of recanalization and reflux in deep veins can be assessed at the earliest after half a year, but since further changes are to be expected up until then, a follow-up 1 yearpostthrombosis seems to be more suitable. Thus, it is our conclusion that a valid statement concerning the prognosis of PTS can be made no earlier than 6 to 12 months post-DVT. It is not uncommon to find patients with advanced CVI without a history of DVT in the phlebological consultation. ${ }^{13}$ However, these patients still show a significant obstruction or reflux in the deep venous system. In these cases, one must presume that an unnoticed DVT occurred in the past. Therefore, therapeutic proceedings are identical for those patients with a known history of DVT. In each case, the indication for anticoagulation must be assessed individually, since it is mostly not necessary to anticoagulate patients.

\section{Compression Therapy}

In the case of acute DVT with strong edema, we recommend initial compression bandaging for primary decongestion, since customized compression stockings usually become too wide once the initial edema has been sufficiently decompressed.

Table 2 CEAP-classification

\begin{tabular}{|l|l|l|l|}
\hline C-Clinical classification & Etiologic classification & Anatomical classification & Pathophysiology \\
\hline $\begin{array}{l}\text { C0: no visible sign of } \\
\text { venous disease }\end{array}$ & Ec: congenital (PTS) & As: superficial & Pr: reflux (R) \\
\hline $\begin{array}{l}\text { C1: telangiectasias } \\
\text { C2: varicular veins }\end{array}$ & Ep: primary (PTS) veins & Ap: deep & Po: obstruction (O) \\
\hline C3: edema & Es: secondary (PTS) & Ad: perforator & PrO: R and O \\
\hline C4a: pigmentation or eczema & $\begin{array}{l}\text { En: no venous } \\
\text { cause identified }\end{array}$ & $\begin{array}{l}\text { An: no venous } \\
\text { location identified }\end{array}$ & $\begin{array}{l}\text { Pnothophysiology identified } \\
\text { p4b: lipodermatosclerosis } \\
\text { or atrophie blanche }\end{array}$ \\
\hline C5: healed ulcer & & & \\
\hline C6: active ulcer & & & \\
\hline S: symptomatic & & & \\
\hline A: asymptomatic & & & \\
\hline
\end{tabular}

Abbreviation: PTS, postthrombotic syndrome. 
Once a distinct reduction of the edema can be seen, we recommend to switch to compression stockings as early as possible, as this leads to better treatment results due to better compliance. If the initial edema is only mild or moderate, stockings can be used immediately. The sole compression of the lower leg using compression stockings, e.g., compression class II A-D, is pathophysiologically sufficient since the calf muscle pump and its support are responsible for approximately $90 \%$ of venous return. ${ }^{14,15}$ Thus, lower leg compression in the follow-up treatment is almost always sufficient for prophylaxis and therapy of PTS. ${ }^{16,17}$

The choice of compression class depends on the prognosis and, above all, the circumference of the lower leg. For sizeable calves, an increase to compression class III is advisable, as the pre-existing tension reduces contact pressure according to the law of Laplace. ${ }^{16,18}$ However, in our professional opinion, class II compression stockings are generally sufficient.

Unfortunately, the success of compression therapy depends predominantly on patient compliance.For patients who are unable to put on class II compression stockings without the help of compression garment donning aids, class I compression stockings should be used instead if manageable. An alternative would be the installation of a nursing service for further assistance.

Nowadays compression stocking manufacturers offer different donning tools, which often work very well. Therefore, it makes sense to guide the patient toward these options. Even in the presence of a florid leg ulcer, compression stockings intended for this purpose are generally superior to bandaging. ${ }^{19,20}$

These compression stockings are usually double-layer stockings consisting of a class I compression stocking as well as a class II stocking top-layer. When combined, this results in class III compression. The undergarment sock fixes the wound dressing and can be worn overnight. These stockings have a significantly lower restriction of ankle mobility, which prevents ankylosis of the ankle joint. For the purpose of achieving optimal compliance, a sufficient analgetic therapy is important.

As an alternative to compression stockings, there are calfbandaging systems with Velcro (adaptive compression sys- tems) and durable compression bandages of several layers. Even so, these permanent dressings severely limit the evaluation of ulcers and are much more common in other health care systems than in the German health care system.

\section{Duplex Sonography}

Two criteria play a major role in duplex sonographic assessment of PTS: recanalization and reflux in the area of the affected veins.

It has been proven useful to divide the degree of recanalization of the deep leg veins into three categories: poorly, moderately, and well recanalized.

Reflux is measured by the velocity of reflux during the Valsalva maneuver while lying down. Reflux rates of up to $0.3 \mathrm{~m} / \mathrm{s}$ are often associated with relative deep vein insufficiency due to varicose veins. Higher reflux rates are usually associated with a PTS. However, to diagnose deep vein insufficiency in addition to reflux velocity, one must always take the appearance of the veins using the B-mode as well as their recanalization into consideration. In our opinion, beside proximal thrombosis, the most unfavorable prognostic factor is a good recanalization of the deep venous system with considerable reflux, especially with regards to the popliteal vein. As a result, high pressures and large blood volumes affect the lower leg and lead to a massively increased pressure within the deep venous system. This combination often rapidly leads to a pronounced PTS, including leg ulcers. Depending on the degree of recanalization and reflux as well as the possible presence of primary or secondary varicose veins, there are several promising treatment options.

In - Fig. 1 we try to establish a reference system for duplex sonographic findings of deep and superficial veins to make therapy decisions easier.

\section{Deep Veins with Reflux or Obstruction without Varicosis}

In this finding, the damage lies exclusively in the area of the deep venous system. Here, the only treatment option is basic compression therapy.

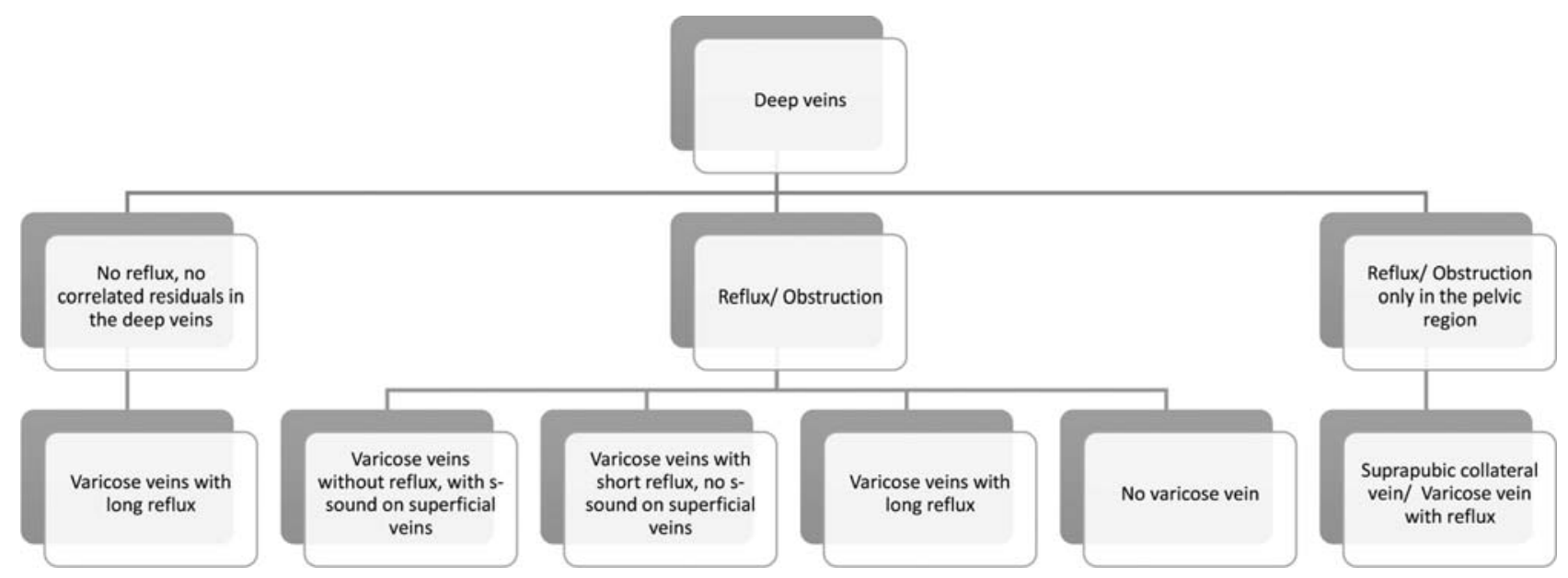

Fig. 1 Schematic of duplex sonographic findings in superficial and deep veins after DVT combined with findings in the deep and superficial leg venous systems. S-sound: spontaneous flow. DVT, deepvein thrombosis. 
In the case of leg ulcers, in addition to moist wound treatment the option of reconstructing tissue defects using shaving methods and mesh graft skin transplantation as well as a vacuum pump system should be considered.

\section{Deep Veins without Reflux or Residual Varicosis with Long Reflux}

If the deep venous system does not show any measurable reflux or residual venous thrombosis in the sense of obstruction, concomitant CVI is not the direct result of thrombosis. In this case, varicosis with long reflux should be classified as primary varicosis, which usually developed prior to thrombosis. In this instance, varicose vein eliminating therapy is indicated. Nevertheless, endovenous treatment options should be used cautiously, as these are more prone to postoperative thrombosis than conventional, modern varicose vein surgery using stripping and exhairese. Surgical intervention is possible even with a florid ulcus cruris, although it is currently being discussed whether the healing time of leg ulcers depends on whether or not a varicose vein surgery is performed in addition to compression therapy. ${ }^{21,22}$ In any case, varix-interrupting therapy is generally indicated for relapse prevention of leg ulcers. With regards to surgical treatment of PTS, prolonged thrombosis prophylaxis should always be the goal due to an increased risk of rethrombosis.

\section{Deep Veins with Reflux or Obstruction- Varicosis with Long Reflux}

If PTS is accompanied by varicose veins with prolonged reflux, it is usually advisable to eliminate varicose veins surgically to improve hemodynamics. In particular, surgical treatment of the small saphenous vein is always a treatment option in this constellation. However, the surgical removal of the great saphenous vein also, especially with existing florid leg ulcers, leads to much faster healing of the existing ulcer according to our experience.

\section{Deep Veins with Reflux or Obstruction- Varicosis with Short Reflux without S-Sound}

If PTS with concomitant varicose veins shows short reflux but no spontaneous flow (s-sound), it is not possible to clearly predict from the duplex finding whether a varicose vein surgery is profitable for the patient. In addition, phlebodynamometry should be performed to assess more accurately whether the hemodynamics can be improved in the affected area by varix-interrupting measures. Should this not be confirmed, consistent compression therapy is the only therapeutic option. Other methods of measuring the hemodynamic status of the leg to predict the operative outcome such as digital photoplethysmography ultimately do not present as satisfactory alternatives, because of preexisting skin damages, which interfere with the use of such methods.

\section{Deep Veins with Reflux or Obstruction- Varicosis without Reflux with S-Sound}

Varicosis without measurable reflux in existing PTS and with existing s-sound is to be regarded as a bypass circuit and thus as secondary varicosis, which should be treated primarily by means of compression therapy. For patients presenting with leg ulcers too, a surgical therapy in the area of the ulcer as well as a varicose vein surgery in the area surrounding the ulcer may be considered. In particular, foam sclerotherapy of the affected veins by means of $1 \%$ foamed polidocanol is an effective treatment option. This can also be combined with a varicose vein surgery. A phlebodynamometric examination may additionally be performed to predict the success of this treatment option (-Figs. 2 and 3).

\section{Isolated Obstruction or Reflux in the Pelvic Floor with Spontaneous Palma- Varicosiswith Reflux}

This condition is usually found after a pelvic vein thrombosis of the iliac vein or a DVT of the leg. Due to poor visibility in the pelvic area with duplex sonography, diminished respiratory modulation on the common femoral vein is an indirect indicator. A varicose vein surgery distally of the saphenous junction is usually beneficial. In this case as well, surgical treatment of the small saphenous vein is always an option. Here, the focus lies on the risk of thrombosis of the suprapubic collateral veins ("spontaneous palma") or its region of drainage when performing a crossectomy on the contralateral leg. If there is no measurable flow on the suprapubic varices, the exhairesis of the "spontaneous palma" should be the primary goal of varicose vein surgery. To what extent a stent of the pelvic floor can contribute to the improvement of hemodynamics, e.g., in pelvic vein thrombosis, still remains unclear, but it can be considered in individual cases.

\section{Local Therapy of Leg Ulcer}

In advanced PTS, an adequate therapy of the leg ulcer plays a crucial role. The healthindustry provides a confusing variety of therapeutic wound dressings. For most of them no evidence-based advantages in terms of healing tendency of leg ulcers have been demonstrated. The advantage of moist wound treatment however is undisputed. ${ }^{23,24}$ As a result, a small portfolio of wound dressings in the treatment of leg ulcers is generally sufficient to adequately treat all patients. A stage-adapted wound therapy should always be the primary therapeutic goal (-Fig. 4).

\section{Wound Gauze}

Wound gauze prevents adherence of the wound dressing to the wound itself. A modern wound gauze with a low Vaseline content should be chosen. Alternatively, it is possible to use a silicone grid. However, silicone grids are more expensive and offer insufficient benefits in comparison to the use of gauze. 


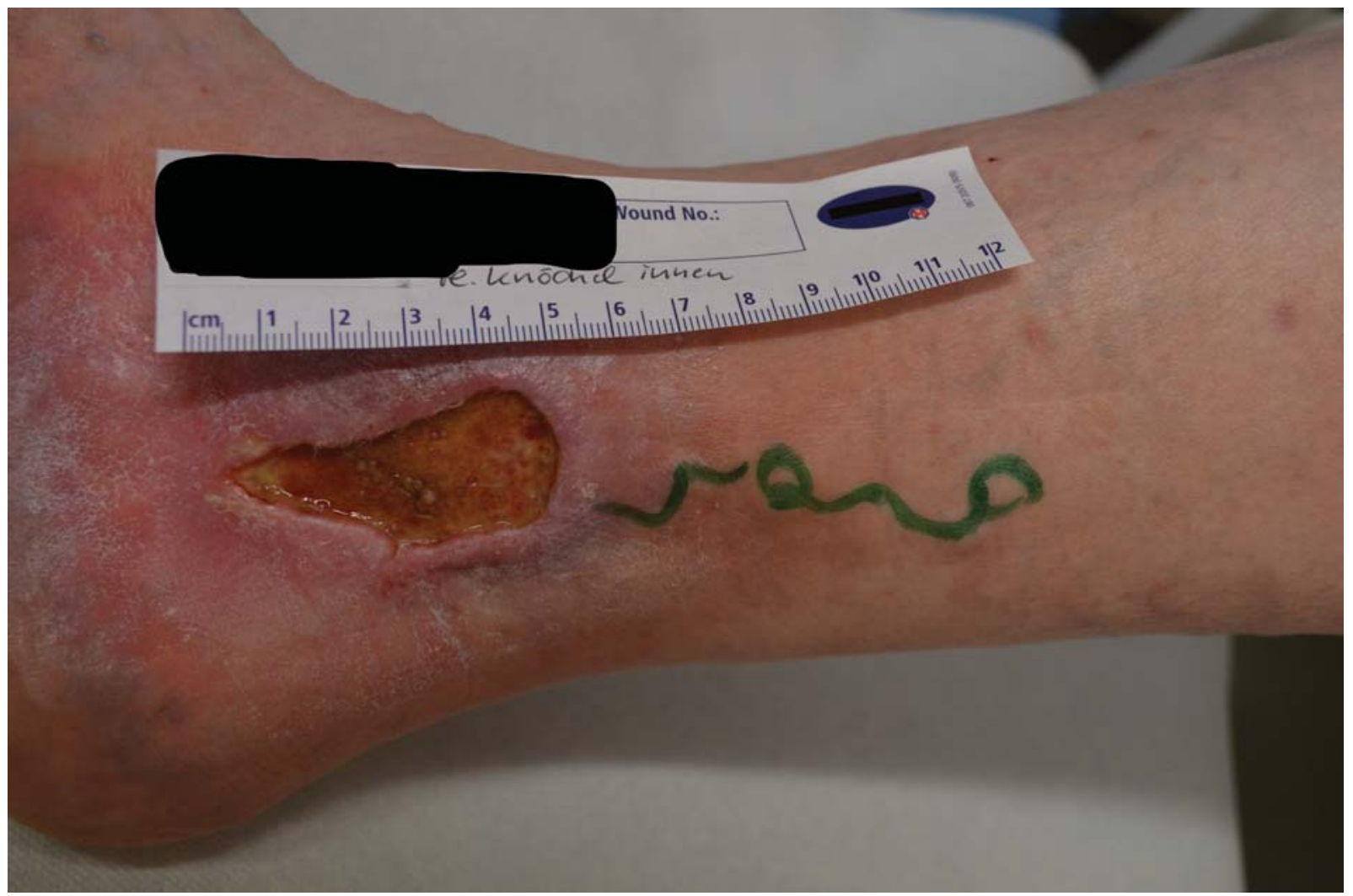

Fig. 2 Recurrent ulcer in severe PTS over decades without healing tendency under multimodal conservative therapy for more than a year. Proximal of the ulcer insufficient posterior tibial perforator vein (Cockett) perforator with nutrient vein. The remaining venous system did not show any signs of reflux. PTS, postthrombotic syndrome.

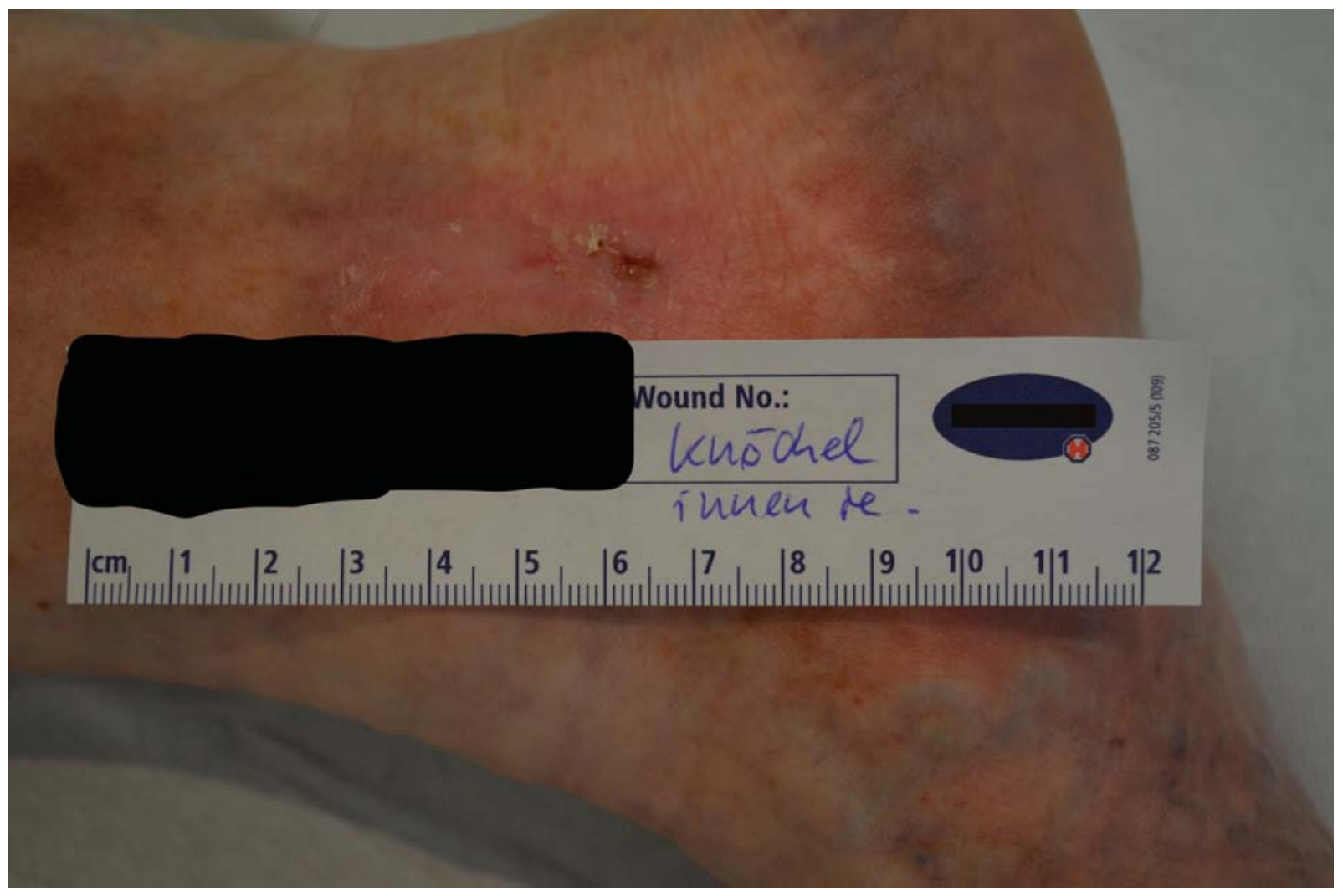

Fig. 3 After ligature of the perforator and exhairesis of the proximal venous vein, as well as foam sclerosis of the peri-ulcerous veins, the ulcer has regressed completely, relapse free to this moment. 


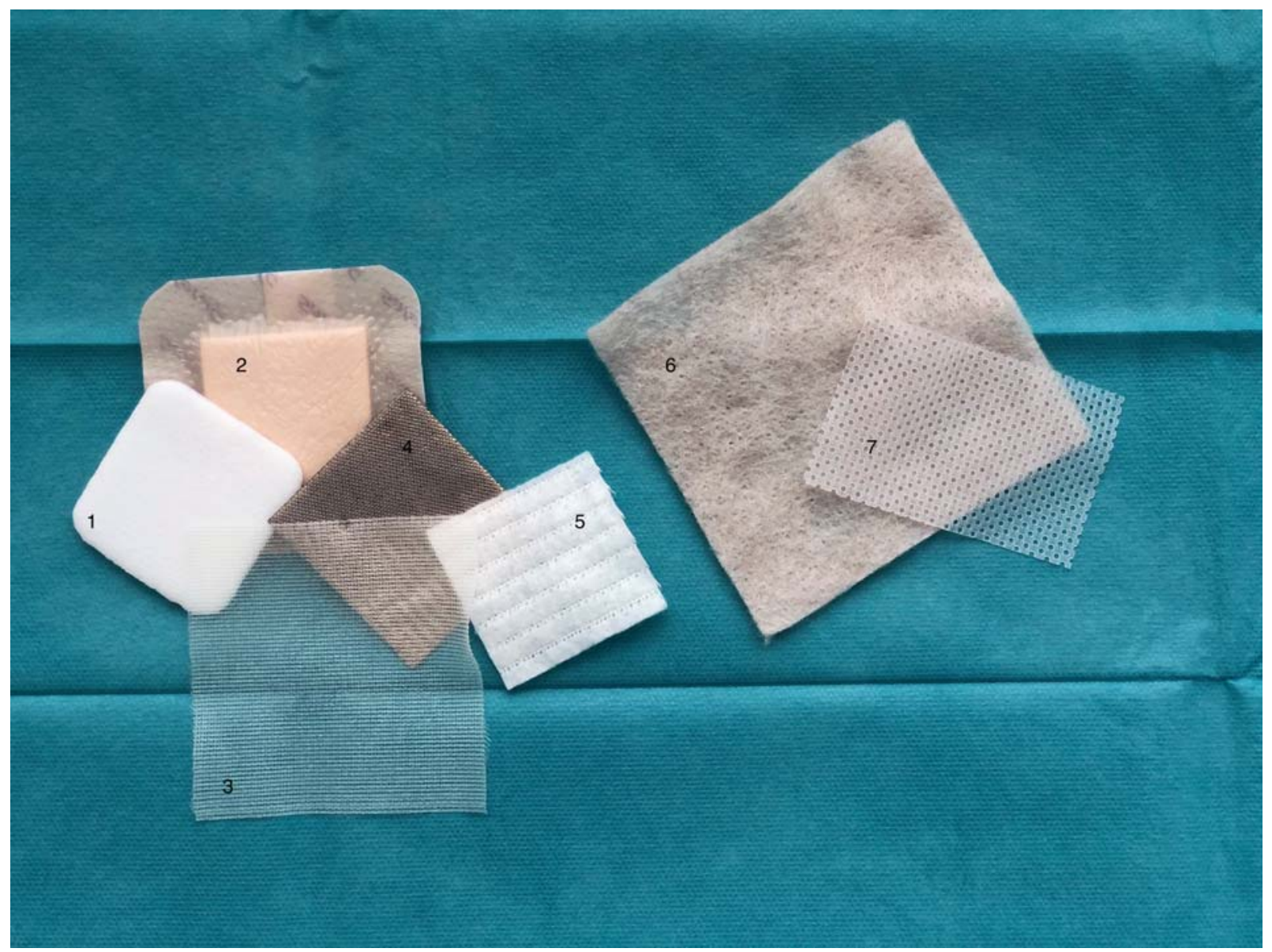

Fig. 4 Minimized wound dressing program to reduce storage costs. 1 and 2: Polyurethane foam with and without an adhesive border. 3: Wound gauze. 4: Dressing containing activated carbon and silver. 5:Hydrofiber wound dressing. 6: Alginate dressing. 7: Silicone wound contact layer.

\section{Wound Covers Adapted to the Disease Stage and Exudate Management}

In principle, throughout all stages of wound healing, application of closed-cell polyurethane foam is desirable. This effectuates covering of the ulcer and provides a very good management of exudation. Furthermore, a special coating of the closed-cell polyurethane foam can lead to a faster wound healing. Depending on the nature of the wound margin, it can be used with or without an adhesive rim. ${ }^{25,26}$ The benefit of adding silver to wound dressings has not yet been proven, since wound dressings generally do not release sufficiently high silver-ion concentrations into the wound to achieve an antibacterial effect.

\section{Wound Moisturizing}

Frequently wounds become too dry, but fortunately there are several options to moisturize the wound. Of course, wound gels that are being offered specifically for this purpose can be used. Alternatively, moistened alginate or a hydrofiber can be applied to moisten the wound. This way, keeping hydrogels in stock becomes unnecessary.

\section{Antiseptic Therapy}

Systemic antibiotic therapy does not usually play a role in the therapy of leg ulcers. Local antibiotic treatment is also considered obsolete. However, in the case of acute systemic signs of infection, systemic antibiotic therapy may be indi- cated in individual cases. In principle, it is possible to reduce germ colonization with the sole use of antiseptic therapy, where polyhexanides or biguanides take the lead. These can easily be applied as a gel which remains on the ulcer for a longer time. Due to water-based galenics, they have the advantage that they do not sting when applied to the wound.

After varicose vein treatment in the presence of a florid leg ulcer, there is always a risk of wound infection. Therefore, the administration of a perioperative single-shot antibiotic is favorable for the patient. In addition, either an antiseptic gel or a wound dressing with a high proportion of silver for infection prophylaxis can be used to reduce germ colonization in the area of acute leg ulcer. For this purpose however, only one product with activated carbon combined with silver, which releases a sufficiently high silver-ion concentration into the wound, is available. This combined wound dressing has the further advantage that the activated carbon minimizes leakage of infectious exudate.

\section{Protection of Wound Surroundings}

Frequently, wound surroundings are affected by moist wound treatment. Not approved for this indication, but highly effective and extremely friendly to the skin, is a phenylmethyl silicone and hexamethyldisiloxane-containing skin protection film. This originates from stoma care and can easily be applied using a lollipop applicator. 
By limiting substance variety, storage and training of new staff can be greatly facilitated without degradation in patient care. At the same time, this reduces the cost of wound care.

\section{Conflict of Interest}

The authors declare that they have no conflict of interest.

\section{References}

1 Palacios FS, Rathbun SW. Medical treatment for postthrombotic syndrome. SeminInterventRadiol 2017;34(01):61-67

2 Kahn SR. The post-thrombotic syndrome. Hematology (Am Soc Hematol Educ Program) 2016;2016(01):413-418

3 Galanaud JP, Monreal M, Kahn SR. Epidemiology of the postthrombotic syndrome. Thromb Res 2018;164:100-109

4 Hach-Wunderle V, Bauersachs R, Gerlach HE, et al. Post-thrombotic syndrome 3 years after deep venous thrombosis in the Thrombosis and Pulmonary Embolism in Out-Patients (TULIPA) PLUS Registry. J Vasc Surg Venous LymphatDisord 2013;1(01):5-12

5 Rabinovich A, Kahn SR. How to predict and diagnose postthrombotic syndrome. Pol Arch Med Wewn 2014;124(7-8):410-416

6 Soosainathan A, Moore HM, Gohel MS, Davies AH. Scoring systems for the post-thrombotic syndrome.J Vasc Surg 2013;57(01):254-261

7 Vedantham S, Goldhaber SZ, Julian JA, et al; ATTRACT Trial Investigators. Pharmacomechanicalcatheter-directed thrombolysis for deep-vein thrombosis. N Engl J Med 2017;377(23): 2240-2252

8 Stain M, Schönauer V, Minar E, et al. The post-thrombotic syndrome: risk factors and impact on the course of thrombotic disease. J ThrombHaemost 2005;3(12):2671-2676

9 Rabe E, Pannier-Fischer F, Bromen K, et al. Bonner Venenstudie der Deutschen Gesellschaft für Phlebologie. Phlebologie 2003;32(01): 1-14

10 Kahn SR, Shapiro S, Wells PS, et al; SOX trial investigators. Compression stockings to prevent post-thrombotic syndrome: a randomised placebo-controlled trial. Lancet 2014;383(9920):880-888

11 Rabinovich A, Ducruet T, Kahn SR; SOX Trial Investigators. Development of a clinical prediction model for the postthrombotic syndrome in a prospective cohort of patients with proximal deep vein thrombosis. J ThrombHaemost 2018;16(02):262-270

12 Jalaie $\mathrm{H}$, Schleimer $\mathrm{K}$, Barbati ME, et al. Interventional treatment of postthrombotic syndrome. Gefasschirurgie 2016;21(Suppl 2):37-44

13 Malkani RH, Karia R, Thadani S. A study of risk factors of chronic venous insufficiency and its association with features suggestive of preceding or present deep venous thrombosis. Indian J Dermatol 2019;64(05):366-371
14 Heinen MM, van Achterberg T, op Reimer WS, van de Kerkhof PC, de Laat E. Venous leg ulcer patients: a review of the literature on lifestyle and pain-related interventions. J Clin Nurs 2004;13(03): 355-366

15 Williams KJ, Ayekoloye O, Moore HM, Davies AH. The calf muscle pump revisited. J Vasc Surg Venous LymphatDisord 2014;2(03): 329-334

16 Lee BB, Nicolaides AN, Myers K, et al. Venous hemodynamic changes in lower limb venous disease: the UIP consensus according to scientific evidence. IntAngiol 2016;35(03):236-352

17 Azirar S, Appelen D, Prins MH, Neumann MH, de Feiter AN, Kolbach DN. Compression therapy for treating post-thrombotic syndrome. Cochrane Database Syst Rev 2019;9:CD004177

18 Prandoni P, Lensing AW, Prins MH, et al. Below-knee elastic compression stockings to prevent the post-thrombotic syndrome: a randomized, controlled trial. Ann Intern Med 2004; 141(04):249-256

19 Blecken SR, Villavicencio JL, Kao TC. Comparison of elastic versus nonelastic compression in bilateral venous ulcers: a randomized trial. J Vasc Surg 2005;42(06):1150-1155

20 Jünger M, Wollina U, Kohnen R, Rabe E. Efficacy and tolerability of an ulcer compression stocking for therapy of chronic venous ulcer compared with a below-knee compression bandage: results from a prospective, randomized, multicentre trial. Curr Med Res Opin 2004;20(10):1613-1623

21 Gohel MS, Barwell JR, Taylor M, et al. Long term results of compression therapy alone versus compression plus surgery in chronic venous ulceration (ESCHAR): randomised controlled trial. BMJ 2007;335(7610):83

22 Barwell JR, Davies CE, Deacon J, et al. Comparison of surgery and compression with compression alone in chronic venous ulceration (ESCHAR study): randomised controlled trial. Lancet 2004; 363(9424):1854-1859

23 Metzger S. Clinical and financial advantages of moist wound management. Home Healthc Nurse 2004;22(09):586-590

24 Jones J. Winter's concept of moist wound healing: a review of the evidence and impact on clinical practice. J Wound Care 2005;14 (06):273-276

25 Meaume S, Truchetet F, Cambazard F, et al; CHALLENGE Study Group. A randomized, controlled, double-blind prospective trial with a Lipido-Colloid Technology-Nano-OligoSaccharide Factor wound dressing in the local management of venous leg ulcers. Wound Repair Regen 2012;20(04):500-511

26 Sigal ML, Addala A, Maillard H, et al. Evaluation of TLC-NOSF dressing with poly-absorbent fibres in exuding leg ulcers: two multicentric, single-arm, prospective, open-label clinical trials. J Wound Care 2019;28(03):164-175 